\title{
DISH AS A CAUSE OF DYSPHAGIA
}

Raissa Velasques de Figueiredo 1,", Maria Lucia Lemos Lopes ${ }^{1}$, Tatiana Freitas Tourinho ${ }^{1}$, Maria Odete Esteves Hilario ${ }^{1}$, Eduardo Rosa de Oliveira ${ }^{1}$, Thiago Willers ${ }^{1}$, Rafael Coradin ${ }^{1}$, Gilberto Scanagatta ${ }^{1}$, Bruno Trevisan ${ }^{1}$, Gabriela Sasso Padilha ${ }^{1}$, Luana Ribeiro Carlos ${ }^{1}$

1. Universidade Federal de Ciências da Saúde de Porto Alegre, Porto Alegre (RS), Brazil.

*Corresponding author: consultoriowillers@gmail.com

\section{BACKGROUND}

Diffuse idiopathic skeletal hyperostosis (DISH), formerly known as Forestier's disease, is characterized by bone neoformation that can affect the axial and peripheral skeleton. Its pathogen is still unknown, but it is associated with male gender, advanced age, obesity, hypertension, atherosclerosis and diabetes mellitus.

\section{CASE REPORT}

A 70-year-old patient, hypertensive, diabetic, dyslipidemic and ex-smoker has been referred to the tertiary hospital due to axial stiffness and mechanical pain. No previous history of inflammatory low back pain, uveitis or family history of psoriasis. The patient denied peripheral arthritis. Laboratories showed negative antinuclear antibody (ANA), negative rheumatoid factor, negative HLA B27. There was no involvement of the sacroiliac joint on radiography. In spine radiography, marked kyphosis, osteophytes and formation of L3-L4 interspinal bone bridges were observed. During physical examination, an important limitation of mobility of the cervical spine was observed, with limitation of extension, flexion and lateral rotation. Schober's test $13 \mathrm{~cm}$. During anamnesis, the patient reports having significant difficulty in swallowing which causes important dietary limitations. He mentioned multiple treatments for gastroesophageal reflux diseases. He also reported having been diagnosed with obstructive sleep apnea, using previous CPAP. Radiography of the cervical region was requested (Fig. 1), and important ossification of the anterior longitudinal ligament was observed. When reviewing previous exams, we observed a deglutogram video with a description of an important anterior osteophyte between C2-C5 that determined compression and ventral displacement of the pharynx obstructing the passage of solid foods. Analyzing the clinical condition in conjunction with radiographic examinations of the patient, we considered the case as DISH and referred the patient to the orthopedist for evaluation of the procedure in the cervical spine with a view to limiting his quality of life by compromising his diet and sleep pattern.

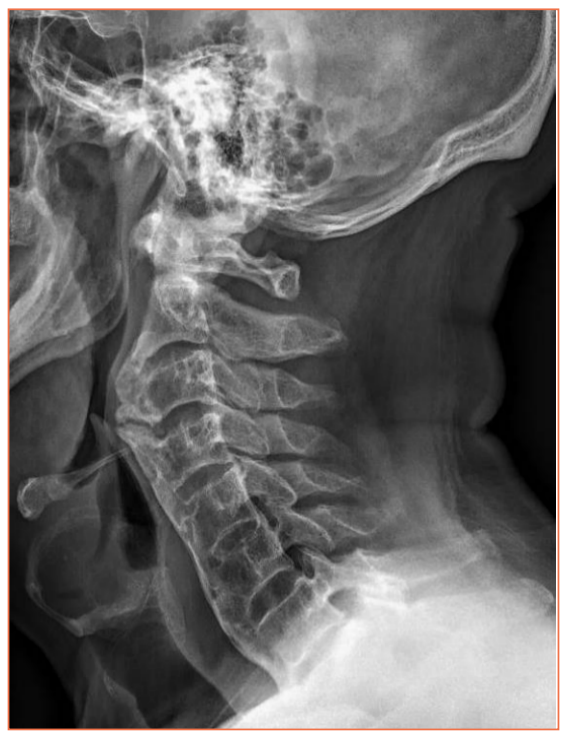

Figure 1. Radiography of the cervical region. 


\section{CONCLUSION}

Diffuse idiopathic skeletal hyperostosis is a long-forgotten, but not-so-rare cause of dysphagia. It is important, therefore, that we do not forget to make an evaluation of the cervical spine in these patients with important complaints where other causes are not found, especially when the patient fits the epidemiological pattern of the disease. 